\title{
Mean Square Deviation Analysis of LMS and NLMS Algorithms with White Reference Inputs
}

\author{
Sheng Zhang, Jiashu Zhang, Hing Cheung So, EURASIP Member
}

\begin{abstract}
This paper investigates the mean square performance of the least mean square (LMS) and normalized LMS (NLMS) algorithms with white reference inputs. Their closed-form mean square deviation (MSD) expressions for the transient and steady-state regimes are derived. Additionally, bounds on the step-size which guarantee mean square stability are given. It is found that the step-size bound and transient behavior of the LMS and the steadystate MSD of the NLMS depend on the kurtosis of the input signal. Convergence rates and steady-state MSDs of the two algorithms are then compared, which shows that the normalized variant with a large step-size would offer faster convergence rate than the LMS scheme. However, when small step-sizes are employed, the LMS achieves lower steady-state MSD than the NLMS at the same convergence rate.
\end{abstract}

\section{Index Terms}

LMS, NLMS, step-size bound, steady-state, kurtosis.

\section{INTRODUCTION}

Adaptive filtering has attracted much research interest in both theoretical and applied aspects for a long time [1]-[3]. Due to the good performance and easy implementation, the least mean square (LMS) and normalized LMS (NLMS) algorithms have been widely used in various applications [4]-[7].

There have been numerous works [8]-[20] on analyzing the performance of the LMS and NLMS algorithms for Gaussian inputs. In [9]-[11], the mean and mean square behaviors of the LMS algorithm for Gaussian inputs were studied with the use of the fourth-order Gaussian moment. When the input is stationary and zero-mean Gaussian distributed, the convergence performance of the NLMS algorithm had been presented in [14]-[16], which shows that this method exhibits improved convergence rate in the mean, but at the expense of high steady-state error. In these analyses, the independence assumption was used. The comprehensive study of the independence theory results were obtained by Gardner in [17]. Assuming sufficiently small step-size conditions, the steady-state behavior

This work was partially supported by National Science Foundation of China (Grant: 61271341).

S. Zhang and J. Zhang are with the Sichuan Province Key Lab of Signal and Information Processing at Southwest Jiaotong University, Chengdu, 610031, China (e-mail: 604266280@qq.com, jszhang@home.swjtu.edu.cn).

H. C. So is with the Department of Electronic Engineering, City University of Hong Kong, Kowloon, Hong Kong (e-mail: hcso@ee.cityu.edu.hk). 
of the LMS algorithm was presented in [18], where the independence assumption is not required. In [19]-[21], closed-form expressions for the transient behaviors and the steady-state excess mean square error of the LMS and NLMS were developed. When all signals are bounded, new upper bound for the step-size of LMS algorithm was proposed in [22]. In addition, their mean square performance for cyclostationary white Gaussian input signals had been studied in [23]. However, the characteristics of the LMS and NLMS algorithms for non-Gaussian inputs have not been well studied in the literature.

In this paper, we study the mean square behavior of the LMS and NLMS algorithms in the context of adaptive noise canceling [24], [25] for different white reference input distributions. It points out the effect of the kurtosis of the reference input signal on their convergence performance. Specifically, the analysis is done in the context of tracking a first-order Markov plant, which is a random walk to model a non-stationary signal for the optimum weights [6], [19], [23]. Mathematical models are derived for the mean square behavior of the LMS and NLMS. Their step-size bounds and steady-state behaviors are also produced. Simulation results are performed to validate our theoretical development.

\section{SYSTEM MODEL}

Consider the adaptive noise cancellation application shown in Fig. 1, where $x(n)$ is the reference input, and $s(n)$ is the desired signal. The primary input $d(n)$ to the canceller is $d(n)=\mathbf{w}^{T}(n) \mathbf{x}(n)+s(n)$, where $\mathbf{x}(n)=$ $[x(n), x(n-1), \cdots, x(n-L+1)]^{T}, \mathbf{w}(n)=\left[w_{1}(n), w_{2}(n), \cdots, w_{L}(n)\right]^{T}$, and $(\cdot)^{T}$ is the transpose of $(\cdot)$. The canceller output is $e(n)=d(n)-\hat{\mathbf{w}}^{T}(n) \mathbf{x}(n)$, where $\hat{\mathbf{w}}(n)=\left[\hat{w}_{1}(n), \hat{w}_{2}(n), \cdots, \hat{w}_{L}(n)\right]^{T}$ contains the adaptive filter tap weights at time $n$. The unknown coefficient vector in the context of a non-stationary system is a first-order Markov model $\mathbf{w}(n+1)=\mathbf{w}(n)+\mathbf{q}(n)$, where $\mathbf{q}(n)$ denotes the random perturbation.

The $\operatorname{MSD}$ is $\operatorname{MSD}(n)=\operatorname{Tr}\left(E\left\{(\mathbf{w}(n)-\hat{\mathbf{w}}(n))(\mathbf{w}(n)-\hat{\mathbf{w}}(n))^{T}\right\}\right)$, where $\operatorname{Tr}(\cdot)$ is the trace of a matrix and $E\{\cdot\}$ is the mathematical expectation of a random variable.

To keep the calculations mathematically tractable, the following assumptions are adopted for the LMS and NLMS algorithms.

Assumption I: $\{x(n)\}$ is an i.i.d. stationary sequence with even probability density function, finite variance $\sigma_{x}^{2}$, finite $E\left\{x^{4}(n)\right\}$, and finite $E\left\{1 /\left(\mathbf{x}^{T}(n) \mathbf{x}(n)\right)\right\}$. The kurtosis $\kappa$ of $x(n)$ is $\kappa=\frac{E\left\{x^{4}(n)\right\}}{\left[E\left\{x^{2}(n)\right\}\right]^{2}}$. In addition, the existence of $E\left\{x^{4}(n)\right\}$ and $E\left\{1 /\left(\mathbf{x}^{T}(n) \mathbf{x}(n)\right)\right\}$ is needed for the LMS and NLMS algorithms, respectively. This is because the non-existence of $E\left\{x^{4}(n)\right\}$ and $E\left\{1 /\left(\mathbf{x}^{T}(n) \mathbf{x}(n)\right)\right\}$ will lead to divergence of the LMS and NLMS in the mean square sense [26].

Assumption II: $\{s(n)\}$ is i.i.d. with zero-mean and finite variance $\sigma_{s}^{2}$, and is independent of $\mathbf{x}(n)$ for all $n$.

Assumption III: The random perturbation vector $\mathbf{q}(n)$ is stationarly i.i.d. zero-mean vectors with $E\left\{\mathbf{q}(n) \mathbf{q}^{T}(n)\right\}=$ $\sigma_{q}^{2} \mathbf{I}$, and is mutually independent of $\mathbf{x}(n)$ and $s(n)$.

Assumption IV: $\hat{\mathbf{w}}(n)$ is independent of $\mathbf{x}(n)$, which is valid when the step-size is sufficiently small [19]-[21]. 


\section{MEAN SQUARE ANALYSIS OF LMS FOR WHITE REFERENCE INPUTS}

The LMS weight update recursion is

$$
\hat{\mathbf{w}}(n+1)=\hat{\mathbf{w}}(n)+\mu \mathbf{x}(n) e(n)
$$

where $\mu$ is the step-size. Let $\mathbf{v}(n)=\mathbf{w}(n)-\hat{\mathbf{w}}(n)$ denote the weight error vector, the canceller output is

$$
e(n)=s(n)+\mathbf{v}^{T}(n) \mathbf{x}(n)
$$

and the weight error vector of the LMS can be expressed as

$$
\mathbf{v}(n+1)=\mathbf{v}(n)-\mu \mathbf{x}(n)\left(s(n)+\mathbf{x}^{T}(n) \mathbf{v}(n)\right)+\mathbf{q}(n)
$$

Using Assumptions II-IV and post-multiplying (3) by its transpose, taking expectations yields

$$
\begin{aligned}
& E\left\{\mathbf{v}(n+1) \mathbf{v}^{T}(n+1)\right\}=E\left\{\mathbf{v}(n) \mathbf{v}^{T}(n)\right\}-2 \mu \sigma_{x}^{2} E\left\{\mathbf{v}(n) \mathbf{v}^{T}(n)\right\} \\
& \quad+\mu^{2} E\left\{\mathbf{x}(n) \mathbf{x}^{T}(n) E\left\{\mathbf{v}(n) \mathbf{v}^{T}(n)\right\} \mathbf{x}(n) \mathbf{x}^{T}(n)\right\}+\mu^{2} \sigma_{x}^{2} E\left\{s^{2}(n)\right\} \mathbf{I}+\sigma_{q}^{2} \mathbf{I}
\end{aligned}
$$

Employing Assumption I, the third expected value of the right side of (4) is

$$
E\left\{\mathbf{x}(n) \mathbf{x}^{T}(n) E\left\{\mathbf{v}(n) \mathbf{v}^{T}(n)\right\} \mathbf{x}(n) \mathbf{x}^{T}(n)\right\}=\operatorname{Tr}\left(E\left\{\mathbf{v}(n) \mathbf{v}^{T}(n)\right\}\right) \sigma_{x}^{4} \mathbf{I}+(\kappa-1) \sigma_{x}^{4} E\left\{\mathbf{v}(n) \mathbf{v}^{T}(n)\right\}+\Delta
$$

where $\Delta$ is defined in Appendix and its main diagonal elements are zero. The kurtosis $\kappa$ can never be less than 1, because $E\left\{x^{4}\right\} \geq\left[E\left\{x^{2}\right\}\right]^{2}$ for the random variable $x$, due to Schwartz inequality [28]. Substituting (5) into (4), we obtain

$$
\begin{aligned}
& E\left\{\mathbf{v}(n+1) \mathbf{v}^{T}(n+1)\right\}=E\left\{\mathbf{v}(n) \mathbf{v}^{T}(n)\right\}-2 \mu \sigma_{x}^{2} E\left\{\mathbf{v}(n) \mathbf{v}^{T}(n)\right\} \\
& \quad+\mu^{2} \sigma_{x}^{4}\left(\operatorname{Tr}\left(E\left\{\mathbf{v}(n) \mathbf{v}^{T}(n)\right\}\right) \mathbf{I}+(\kappa-1) E\left\{\mathbf{v}(n) \mathbf{v}^{T}(n)\right\}\right)+\mu^{2} \Delta+\mu^{2} \sigma_{x}^{2} E\left\{s^{2}(n)\right\} \mathbf{I}+\sigma_{q}^{2} \mathbf{I}
\end{aligned}
$$

Taking the trace of both sides of (6) yields

$$
\operatorname{MSD}(n+1)=\left(1-2 \mu \sigma_{x}^{2}+\mu^{2} \sigma_{x}^{4}(L+\kappa-1)\right) \operatorname{MSD}(n)+\mu^{2} \sigma_{x}^{2} \sigma_{s}^{2} L+\sigma_{q}^{2} L
$$

It is shown that for different reference inputs with the same variance, the performance of the LMS is associated with the kurtosis of the reference input. In the following, it will be discussed in more detail.

\subsection{Stability}

The mean square stability requirement based on (7) is $\left|1-2 \mu \sigma_{x}^{2}+\mu^{2} \sigma_{x}^{4}(L+\kappa-1)\right|<1$, i.e., $\mu$ should satisfy:

$$
\mu \in\left(0, \frac{2}{(L+\kappa-1) \sigma_{x}^{2}}\right)
$$

It is known that the selection range of the step-size of the LMS algorithm is strongly dependent on the power of the reference input signal [3]. However, (8) also shows that the step-size bound is also dependent on the kurtosis of the reference input signal.

Remark 1: (i) If there are different inputs with $\kappa_{1}<\kappa_{2}$, we have

$$
\frac{2}{\left(L+\kappa_{2}-1\right) \sigma_{x}^{2}}<\frac{2}{\left(L+\kappa_{1}-1\right) \sigma_{x}^{2}}
$$

(ii) From (7), the transient MSD behavior of the LMS is affected by the factor $1-2 \mu \sigma_{x}^{2}+\mu^{2} \sigma_{x}^{4}(L+\kappa-1)$, which shows that the reference input with large kurtosis leads to slow convergence rate. 


\subsection{Steady-state performance}

Assuming that (7) is operating in steady-state, and (8) is satisfied, we have

$$
\operatorname{MSD}(\propto)=\frac{L \mu \sigma_{s}^{2}}{2-\mu(L+\kappa-1) \sigma_{x}^{2}}+\frac{L \sigma_{q}^{2}}{2 \mu \sigma_{x}^{2}-\mu^{2}(L+\kappa-1) \sigma_{x}^{4}}
$$

which is a monotonically increasing function of $\kappa>0$.

Remark 2: (i) According to (10), the steady-state MSD of the LMS for the white reference input signal is not only controlled by the step-size, the filter length, the powers of desired and reference input signals, but also dependent on the kurtosis of reference input. For small kurtosis $\kappa$ with $\kappa \ll L$, the steady-state MSD is weakly correlated with $\kappa$. On the contrary, if there is a reference input signal with $\kappa \gg L$, the correlation between steadystate MSD and the kurtosis $\kappa$ cannot be ignored. When the step-size is sufficiently small such that $\mu \ll \frac{2}{(L+\kappa-1) \sigma_{x}^{2}}$, the steady-state MSD has little correlation with $\kappa$.

(ii) If the step-size satisfies the condition $\mu \ll \frac{2}{(L+\kappa-1) \sigma_{x}^{2}}$, we have from (10) for the time-varying system that

$$
\operatorname{MSD}(\propto)=\frac{L \mu \sigma_{s}^{2}}{2}+\frac{L \sigma_{q}^{2}}{2 \mu \sigma_{x}^{2}}
$$

From (11), we have the minimum MSD and the corresponding optimum step-size [2]

$$
\begin{aligned}
\operatorname{MSD}_{\min }(\propto) & =\sqrt{\frac{L^{2} \sigma_{s}^{2} \sigma_{q}^{2}}{\sigma_{x}^{2}}} \\
\mu_{\mathrm{opt}} & =\frac{\sigma_{q}}{\sigma_{s} \sigma_{x}}
\end{aligned}
$$

\section{MEAN SQUARE ANALYSIS OF NLMS FOR WHITE REFERENCE INPUT SIGNALS}

The weight update recursion of the NLMS is

$$
\hat{\mathbf{w}}_{1}(n+1)=\hat{\mathbf{w}}_{1}(n)+\mu \mathbf{x}(n) \frac{e_{1}(n)}{\mathbf{x}^{T}(n) \mathbf{x}(n)}
$$

where

$$
e_{1}(n)=d(n)-\mathbf{x}^{T}(n) \hat{\mathbf{w}}_{1}(n)
$$

Let $\mathbf{v}_{1}(n)=\mathbf{w}_{1}(n)-\hat{\mathbf{w}}_{1}(n)$, the weight error vector of the NLMS algorithm can be written as

$$
\mathbf{v}_{1}(n+1)=\mathbf{v}_{1}(n)-\mu \frac{\mathbf{x}(n) \mathbf{x}^{T}(n) \mathbf{v}_{1}(n)}{\mathbf{x}^{T}(n) \mathbf{x}(n)}-\mu \frac{\mathbf{x}(n) s(n)}{\mathbf{x}^{T}(n) \mathbf{x}(n)}+\mathbf{q}(n)
$$

Proceeding in a manner similar to the analysis of the LMS, using Assumptions II and III with $\operatorname{MSD}_{1}(n)=$ $E\left\{\mathbf{v}_{1}^{T}(n) \mathbf{v}_{1}(n)\right\}$ and $\varphi_{x}=E\left\{\frac{1}{\mathbf{x}^{T}(n) \mathbf{X}(n)}\right\}$ yields

$$
\operatorname{MSD}_{1}(n+1)=\operatorname{MSD}_{1}(n)-\mu(2-\mu) E\left\{\frac{\mathbf{v}_{1}^{T}(n) \mathbf{x}(n) \mathbf{x}^{T}(n) \mathbf{v}_{1}(n)}{\mathbf{x}^{T}(n) \mathbf{x}(n)}\right\}+\mu^{2} \sigma_{s}^{2} \varphi_{x}+\sigma_{q}^{2} L
$$

Using Assumptions I and IV, the expected value of the right side of (17) is derived as (See Appendix A in [27]):

$$
E\left\{\frac{\mathbf{v}_{1}^{T}(n) \mathbf{x}(n) \mathbf{x}^{T}(n) \mathbf{v}_{1}(n)}{\mathbf{x}^{T}(n) \mathbf{x}(n)}\right\}=\frac{1}{L} \operatorname{MSD}_{1}(n)
$$

Substituting (18) into (17) yields

$$
\operatorname{MSD}_{1}(n+1)=\left(1-2 \mu \frac{1}{L}+\mu^{2} \frac{1}{L}\right) \operatorname{MSD}_{1}(n)+\mu^{2} E\left\{\frac{1}{\mathbf{x}^{T}(n) \mathbf{x}(n)}\right\} \sigma_{s}^{2}+\sigma_{q}^{2} L
$$




\subsection{Stability}

The mean square stability for (19) requires that the step-size $\mu$ should be chosen such that

$$
\left|1-2 \mu \frac{1}{L}+\mu^{2} \frac{1}{L}\right|<1
$$

Thus, under Assumption IV, a necessary and sufficient condition for the MSD stability of the NLMS is $0<\mu<2$ [2], [13]. Unlike the LMS, the step-size bound of the NLMS is independent of the kurtosis of reference input signal, which is a merited capability that is not possible in the LMS algorithm. Additionally, because of the transient behavior of the MSD for the NLMS determined by the factor $1-2 \mu \frac{1}{L}+\mu^{2} \frac{1}{L}$ in (19), the transient MSD would be independent of the reference input distribution.

\subsection{Steady-state performance}

Assuming that (19) is operating in steady-state, and (20) is satisfied, we have

$$
\operatorname{MSD}_{1}(\propto)=\frac{1}{2-\mu}\left(\mu L \sigma_{s}^{2} \varphi_{x}+\frac{\sigma_{q}^{2} L^{2}}{\mu}\right)
$$

Thus, the steady-state MSD of the NLMS for a white reference input signal is not only controlled by the step-size, the filter length, the power of desired signal, but is also dependent of $\varphi_{x}$.

For a stationary system, (21) simplifies to

$$
\mathrm{MSD}_{1}(\propto)=\frac{1}{2-\mu} \mu L \sigma_{s}^{2} \varphi_{x}
$$

Taking the $10 \log _{10}$ on both sides of (22) yields the MSD in dB:

$$
10 \log _{10}\left(\operatorname{MSD}_{1}(\propto)\right)=\underbrace{10 \log _{10}\left(\frac{\mu L \sigma_{s}^{2}}{2-\mu}\right)}_{A}+\underbrace{10 \log _{10}\left(\varphi_{x}\right)}_{B}
$$

From (23), the steady-state MSD of the NLMS consists of two parts, i.e., $A$ and $B$. The $A$ is determined by the step-size, the filter length, the power of desired signal, whereas $B$ is only determined by $\varphi_{x}$. Even when a small step-size is used for the NLMS, $B$ in the steady-state MSD still persists. Apparently, it is very different from the LMS, because the steady-state MSD of the LMS is determined by the step-size, the filter length, the power of desired signal and is not sensitive to the statistics of reference input.

For the time-varying system, if the step-size satisfies $\mu<<2$, we have from (21) that

$$
\operatorname{MSD}_{1}(\propto)=\frac{1}{2}\left(\mu L \sigma_{s}^{2} \varphi_{x}+\frac{\sigma_{q}^{2} L^{2}}{\mu}\right)
$$

Then, from (24), the minimum MSD of the NLMS and the corresponding optimum step-size are

$$
\begin{gathered}
\operatorname{MSD}_{1, \min }(\propto)=\sqrt{L^{3} \sigma_{s}^{2} \sigma_{q}^{2} \varphi_{x}} \\
\mu_{\mathrm{opt}}=\frac{\sigma_{q} \sqrt{L}}{\sigma_{s} \sqrt{\varphi_{x}}}
\end{gathered}
$$




\section{COMPARISON OF TWO ALGORITHMS}

In this section, we compare the LMS and NLMS algorithms under Assumptions I-IV. Firstly, the comparison of their convergence rates is presented. Secondly, keeping the same convergence rate, we compare their steady-state MSDs. Finally, their tracking capability is contrasted.

\subsection{Convergence rate comparison}

From (7) and (19), the convergence rates of the LMS and NLMS, respectively, depend on their decay factors, which are defined as

$$
\begin{aligned}
& \alpha_{1}=1-2 \mu \sigma_{x}^{2}+\mu^{2} \sigma_{x}^{4}(L+\kappa-1) \\
& \alpha_{2}=1-2 \mu \frac{1}{L}+\mu^{2} \frac{1}{L}
\end{aligned}
$$

Under the step-size conditions (8) and (20),the minimum $\alpha_{1}$ and $\alpha_{2}$ are determined as:

$$
\begin{aligned}
& \alpha_{1, \min }=1-\frac{1}{L+\kappa-1} \\
& \alpha_{2, \min }=1-\frac{1}{L}
\end{aligned}
$$

Thus, we have $\alpha_{2, \min }<\alpha_{1 \text {,min }}$ for $\kappa>1$. This indicates that under Assumptions I-IV, the NLMS algorithm with a large step-size can offer faster convergence compared with the LMS algorithm [13], [15]. A comparison example is displayed in Fig. 2. However, when the filter length is sufficiently large such that $L>>\kappa$, two algorithms have similar optimum convergence rate.

\subsection{Comparison at same convergence rate}

We choose the step-sizes $\mu$ and $\mu_{1}$ for the LMS and NLMS, respectively, which are sufficiently small such that

$$
\begin{aligned}
& \mu \sigma_{x}^{2}(L+\kappa-1) \ll 2 \\
& \mu_{1} \ll 2
\end{aligned}
$$

Then, using (31) and (32), (7) and (19) are approximated in a stationary system as

$$
\begin{gathered}
\operatorname{MSD}(n+1)=\left(1-2 \mu \sigma_{x}^{2}\right) \operatorname{MSD}(n)+\mu^{2} \sigma_{x}^{2} \sigma_{s}^{2} L \\
\operatorname{MSD}_{1}(n+1)=\left(1-2 \mu_{1} \frac{1}{L}\right) \operatorname{MSD}_{1}(n)+\mu_{1}^{2} \varphi_{x} \sigma_{s}^{2}
\end{gathered}
$$

In order to guarantee the same convergence rate in (33) and (35), the step-sizes should have the relationship

$$
\mu=\mu_{1} \frac{1}{\sigma_{x}^{2} L}
$$

Assuming the LMS and NLMS algorithms are operating in steady-state, from (33) and (34), we obtain

$$
\frac{\operatorname{MSD}(\propto)}{\operatorname{MSD}_{1}(\propto)}=\frac{\mu}{\mu_{1} \varphi_{x}}
$$


Substituting (35) into (36) yields:

$$
\frac{\operatorname{MSD}(\propto)}{\operatorname{MSD}_{1}(\propto)}=\frac{1}{L \sigma_{x}^{2} \varphi_{x}}
$$

Using Jensen inequality [28], the expectation in (37) satisfies

$$
E\left\{\frac{1}{\mathbf{x}^{T}(n) \mathbf{x}(n)}\right\} \geq \frac{1}{E\left\{\mathbf{x}^{T}(n) \mathbf{x}(n)\right\}}=\frac{1}{\sigma_{x}^{2} L}
$$

for $\mathbf{x}^{T}(n) \mathbf{x}(n)>0$.

Substituting (38) into (37) yields

$$
\frac{\operatorname{MSD}(\propto)}{\operatorname{MSD}_{1}(\propto)} \leq 1
$$

This result indicates that under small step-size conditions (31) and (32) the LMS algorithm may offer smaller steady-state MSD than the NLMS algorithm at the same convergence rate. From (37), we know the difference of the steady-state MSD between the LMS and NLMS algorithms depends on $\sigma_{x}^{2} L \varphi_{x}$. It is difficult to derive the closed-form expression of $\varphi_{x}$ for general input distribution ${ }^{1}$. When the reference input is a Gaussian signal, $\operatorname{MSD}(\propto) / \operatorname{MSD}_{1}(\propto)=(L-2) / L[29]$.

\subsection{Tracking performance comparison}

Using (12), (25) and (38), the tracking performance of the LMS and NLMS can be compared using:

$$
\frac{\mathrm{MSD}_{\min }(\propto)}{\mathrm{MSD}_{1, \min }(\propto)}=\sqrt{\frac{1}{L \sigma_{x}^{2} \varphi_{x}}} \leq 1
$$

In order to satisfy the conditions $\mu(L+\kappa-1) \sigma_{x}^{2} \ll 2$ and $\mu_{1} \ll 2$ for the optimum step-sizes (13) and (26), we need

$$
\max \left(\frac{(L+\kappa-1) \sigma_{q} \sigma_{x}}{\sigma_{s}}, \frac{\sigma_{q} \sqrt{L}}{\sigma_{s} \sqrt{\varphi_{x}}}\right) \ll 2
$$

which is the condition of slow variations. This indicates that under the condition (41) the minimum achievable value of steady-state MSD of the LMS algorithm may be less than that of the NLMS algorithm.

Finally, according to the advantage in the LMS and NLMS algorithms, the applicability of two algorithms for a stationary system is summarized in Table I. However, because of better tracking capability for the time-varying system, the LMS algorithm may be a better choice than the NLMS algorithm when the condition (41) is satisfied.

\section{Simulation RESUlts}

This section provides a comparison of the theoretical results of the LMS and NLMS algorithms with Monte Carlo simulations, which are done for the case of adaptive noise cancellation. The unknown system to be estimated in all experiments is a finite impulse response filter of length $L$, and the initial weight vector of the unknown

${ }^{1}$ For a specific distribution of reference input, it can be calculated via numerical integration. For example, [30] shows the result for the CG input. 
system is randomly generated and normalized to unit energy. The length of adaptive filter is equal to that of the corresponding unknown channel. The initial weight vector of the adaptive filter is an all zero vector.

We first evaluate Assumption IV by simulations. The following three terms are based on Assumption IV:

$$
\begin{aligned}
& E\left\{\mathbf{v}^{T}(n) \mathbf{x}(n) \mathbf{x}^{T}(n) \mathbf{v}(n)\right\}=\sigma_{x}^{2} \operatorname{MSD}(n) \\
& E\left\{\mathbf{x}^{T}(n) \mathbf{x}(n) \mathbf{x}^{T}(n) \mathbf{v}(n) \mathbf{v}^{T}(n) \mathbf{x}(n)\right\}=L \sigma_{x}^{4} \operatorname{MSD}(n)+(\kappa-1) \sigma_{x}^{4} \operatorname{MSD}(n) \\
& E\left\{\frac{\mathbf{v}^{T}(n) \mathbf{x}(n) \mathbf{x}^{T}(n) \mathbf{v}(n)}{\mathbf{x}^{T}(n) \mathbf{x}(n)}\right\}=\frac{1}{L} \operatorname{MSD}(n)
\end{aligned}
$$

and their trajectories are plotted in Fig. 3, where the reference input is a white Laplace process with unit variance. From Fig. 3, one can see that the Assumption IV is valid in simplifying our expectation analysis, especially for small step-size conditions. However, for large step-size, the assumption becomes less valid in the transient stage, but is still feasible in steady-state.

Figs. 4 to 5 compares the theoretical and simulation results for the contaminated Gaussian (CG) input. The model of CG signal is $x(n)=x_{g}(n)+b(n) x_{b}(n)$, where $b(n)$ is an i.i.d. Bernoulli random sequence whose value is either zero or one, with occurrence probability $P(b(n)=1)=p, x_{g}(n)$ and $x_{b}(n)$ are both i.i.d. zero-mean Gaussian sequences with respective variances $\sigma_{g}^{2}$ and $\sigma_{b}^{2}$. The average power of $x(n)$ is $p \sigma_{b}^{2}+\sigma_{g}^{2}$ and its kurtosis $\kappa$ is $\kappa=\frac{3\left(\sigma_{g}^{4}+p \sigma_{b}^{4}\right)+6 p \sigma_{g}^{2} \sigma_{b}^{2}}{\left(\sigma_{g}^{2}+p \sigma_{b}^{2}\right)^{2}} \cdot \varphi_{x}$ is calculated by ensemble averaging in simulations. The parameters of the CG input with $\kappa=9.75$ and $\kappa=752.25$ are chosen to $\sigma_{g}^{2}=1, \sigma_{b}^{2}=10, p=0.1$ and $\sigma_{g}^{2}=1, \sigma_{b}^{2}=1000, p=0.001$, respectively. As can be seen, the steady-state MSD is larger for $\kappa_{1}$ than that for $\kappa_{2}$ with $\kappa_{1}>\kappa_{2}$.

Fig. 6 compares the performance of LMS and NLMS algorithms at same convergence rate, where the reference input signal is CG signal with $\sigma_{g}^{2}=1, \sigma_{b}^{2}=1000, p=0.001$. As can be seen, the LMS yields a lower steady-state MSD than the NLMS algorithm does while having the same convergence rate.

\section{CONCLUSION}

This paper studies the mean square behavior of the LMS and NLMS algorithms for different white reference inputs in the context of adaptive noise canceling. The unknown system is modeled by the standard random walk model. It is found that the NLMS algorithm has good stability and transient response, which are independent of the reference input distribution. The NLMS can offer faster convergence rate than the LMS. However, the LMS may have better steady-state MSD property than the NLMS at the same convergence rate. Monte Carlo simulations provide strong support for these theoretical results.

\section{APPENDIX}

Let $\mathbf{x}$ be an i.i.d. zero-mean random vector with covariance $E\left\{\mathbf{x x}^{T}\right\}=\sigma_{x}^{2} \mathbf{I}$ and kurtosis $\kappa$, then for any symmetric $\mathbf{B}$ of compatible dimensions it holds that

$$
E\left\{\mathbf{x x}^{T} \mathbf{B} \mathbf{x} \mathbf{x}^{T}\right\}=\operatorname{Tr}(\mathbf{B}) \sigma_{x}^{4} \mathbf{I}+(\kappa-1) \sigma_{x}^{4} \mathbf{B}+\Delta
$$


where $\Delta_{i, j}$ and $b_{i, j}$ denote the $(i, j)$ entry of $\Delta$ and $\mathbf{B}$ with

$$
\begin{aligned}
\Delta_{i, j} & =(3-\kappa) b_{i, j} \sigma_{x}^{4}, i \neq j \\
\Delta_{i, j} & =0, \quad i=j
\end{aligned}
$$

Proof: Let $\mathbf{S}=E\left\{\mathbf{x x}^{T} \mathbf{B} \mathbf{x} \mathbf{x}^{T}\right\}$ and $s_{i, j}$ denote its $(i, j)$ entry. Then,

$$
s_{i, j}=E\left\{x_{i} x_{j} \sum_{m=0}^{L-1} \sum_{n=0}^{L-1} x_{m} b_{m, n} x_{n}\right\}
$$

where $x_{i}$ denotes the $i$ th component of the vector $\mathbf{x}$ and $L$ is the length of the vector $\mathbf{x}$.

When $i=j$, (47) is

$$
s_{i, i}=E\left\{x_{i}^{2} \sum_{m=0}^{L-1} x_{m}^{2} b_{m, m}\right\}=\operatorname{Tr}(\mathbf{B}) \sigma_{x}^{4}+(\kappa-1) \sigma_{x}^{4} b_{i, i}
$$

When $i \neq j$, (47) is

$$
s_{i, j}=\left(b_{i, j}+b_{j, i}\right) E\left\{x_{i}^{2} x_{j}^{2}\right\}=\left(b_{i, j}+b_{j, i}\right) \sigma_{x}^{4}
$$

Using the fact that $\mathbf{B}$ is symmetric, (48) and (49) can be written as (45). When $\kappa=3$, it corresponds to the fourthorder Gaussian moment theorem [2]. Equation (5) is obtained by substituting $\mathbf{x}=\mathbf{x}(n)$, and $\mathbf{B}=E\left\{\mathbf{v}(n) \mathbf{v}^{T}(n)\right\}$ into (45).

\section{REFERENCES}

[1] C.F.N. Cowan and P.M. Grant, Adaptive Filters, Englewood Cliffs, NJ: Prentice-Hall, 1985

[2] S. Haykin, Adaptive Filter Theory, 4th ed. Upper Saddle River, NJ: Prentice-Hall, 2002.

[3] A.H. Sayed, Fundamentals of Adaptive Filtering, New York: Wiley-Interscience, 2003.

[4] S. Qureshi, "Adaptive equalization data transmissions," IEEE Commun, Mag., vol. 20, pp. 2-16, 1982.

[5] S. Elliott, Signal Processing for Active Control, San Diego, CA: Academic, 2001.

[6] S. Gogineni and A. Nehorai, "Tracking capability of the least mean square algorithm: Application to an asynchronous echo canceller," IEEE Trans. Acoust., Speech, Signal Process., vol. ASSP-35, no. 11, pp. 1570-1578, Nov. 1987.

[7] Y. Shi, L. Huang, C. Qian and H.C. So, "Shrinkage linear and widely-linear complex-valued least mean squares algorithms for adaptive beamforming," IEEE Trans. Signal Process., vol. 63, no. 1, pp. 119-131, Jan. 2015.

[8] B. Widrow, J. McCool, M.G. Larimore, and C.R. Johnson, "Stationary and nonstationary learning characteristics of the LMS adaptive filter," Proc. IEEE, vol. 46, pp. 1151-1162, Aug. 1976.

[9] B. Widrow and E. Walach, "On the statistical efficiency of the LMS algorithm with nonstationary inputs," IEEE Trans. Inform. Theory, vol. IT-30, no. 2, pp. 211-221, Mar. 1984.

[10] N. Bershad and O. Macchi, "Adaptive recovery of a chirped sinusoid in noise, Part II: Performance of the LMS algorithm," IEEE Trans. Signal Process., vol. 39, no. 3, pp. 595-602, Mar. 1991.

[11] A. Feuer and E. Weinstein, "Convergence analysis of LMS filters with uncorrelated Gaussian data," IEEE Trans. Acoust., Speech, Signal Process., vol. ASSP-33, pp. 222-230, Feb. 1985.

[12] M. Rupp, “The behavior of LMS and NLMS algorithms in the presence of spherically invariant processes," IEEE Trans. Signal Process., vol. 41, pp. 1149-1160, Mar. 1993.

[13] D.T.M. Slock, "On the convergence behavior of the LMS and the normalized LMS algorithms," IEEE Trans. Signal Process., vol. 41, pp. 2811-2825, Sep. 1993.

[14] M. Tarrab and A. Feuer, "Convergence and performance analysis of the normalized LMS algorithms with uncorrelated Gaussian data," IEEE Trans. Inform. Theory, vol. IT-34, no. 4, pp. 680-691, 1988. 
[15] N. Bershad, "Analysis of the normalized LMS algorithm with Gaussian inputs," IEEE Trans. Acoust., Speech, Signal Process., vol. ASSP-34, pp. 793-806, 1986.

[16] P.E. An, M. Brown, and C.J. Harris, "On the convergence rate performance of the normalized least-mean-square adaptation," IEEE Trans. Signal Process., vol. 8, no. 5, pp. 1211-1214, Sep. 1997.

[17] W. A. Gardner, "Leaming characteristics of stochastic-gradient-descent algorithms: A general study, analysis, and critique," Signal Process., vol. 6, pp. 113-133, 1984.

[18] H. J. Butterweck, "A steady-state analysis of the LMS adaptive algorithm without use of the independence assumption," in Proc. IEEE ICASSP, Detroit, MI, 1995, pp. 1404-1407.

[19] N.R. Yousef and A.H. Sayed, "A unified approach to the steady-state and tracking analyses of adaptive filters," IEEE Trans. Signal Process., vol. 49, no. 2, pp. 314-324, Feb. 2001.

[20] T.Y. Al-Naffouri and A.H. Sayed, “Transient analysis of data normalized adaptive filters," IEEE Trans. Signal Process., vol. 51, no.3, pp. 639-652, 2003.

[21] S. Zhang and J. Zhang, "Transient analysis of zero attracting NLMS algorithms without Gaussian input signal," Signal Process., vol. 97, pp. 100-109, 2014.

[22] D. Bismor, "Extension of LMS stability condition over a wide set of signals, International Journal of Adaptive Control and Signal Processing, vol. 29, pp. 653-670, 2015.

[23] N.J. Bershad, E. Eweda, and J.C.M. Bermudez, "Stochastic analysis of the LMS and NLMS algorithms for cyclostationary white Gaussian inputs,” IEEE Trans. Signal Process., vol. 62, no. 9, pp. 2238-2249, 2014.

[24] E. Eweda, and N.J. Bershad, "Stochastic analysis of a stable normalized least mean fourth algorithm for adaptive noise canceling with a white Gaussian reference," IEEE Trans. Signal Process.,vol. 60, no.12, pp. 6235-6244, 2012.

[25] B. Widrow, J.R. Glover, J. McCool, J. Kaunitz, C.S. Williams, R.H. Hearn, J.R. Zeidler, E. Dong, R.C. Goodlin, "Adaptive noise cancelling: Principles and applications,” Proc. IEEE, vol. 63, no 12, Dec. 1975.

[26] E. Eweda, "A new approach for analyzing the limiting behavior of the normalized LMS algorithm under weak assumptions," Signal Process., vol. 89, no. 11, pp. 2143-2151, 2009.

[27] E. Eweda, "Mean-square stability analysis of a normalized least mean fourth algorithm for a Markov plant," IEEE Trans. Signal Process., vol. 62, no.24, pp. 6245-6553, 2014.

[28] A. Papoulis, Probability, Random Variables, and Stochastic Processes, 3rd ed. New York: McGraw-Hill, 1991.

[29] M.H. Costa and J.C.M. Bermudez, "An improved model for the normalized LMS algorithm with Gaussian inputs and large number of coefficients," in Proc. Int. Conf. Acoust., Speech, Signal Process., Orlando, FL, USA, May 2002, pp. 1385-1388.

[30] S. Koike, "Performance analysis of the normalized LMS algorithm for complex-domain adaptive filters in the presence of impulse noise at filter input," IEICE Trans. Fundam., vol. E89-A, no. 9, pp. 2422-2428, 2006. 
TABLE I

APPLICABILITY OF THE LMS AND NLMS FOR STATIONARY SYSTEM WITH WHITE REFERENCE INPUT.

\begin{tabular}{c|c|c}
\hline \hline & $L \ll \kappa$ & $L \gg \kappa$ \\
\hline unknown $\sigma_{x}^{2}, \kappa$ & $\begin{array}{c}\text { NLMS } \\
\text { (stable) }\end{array}$ & $\begin{array}{c}\text { NLMS } \\
\text { (stable) }\end{array}$ \\
\hline known $\sigma_{x}^{2}, \kappa$ & $\mathrm{NLMS}^{2}, \mathrm{LMS}^{2}$ & $\begin{array}{c}\text { LMS }^{3} \\
\text { (low complexity) }\end{array}$ \\
\hline \hline
\end{tabular}

${ }^{1}$ The algorithm is chosen to guarantee the stability.

${ }^{2}$ In this case, using the NLMS can offer faster optimum convergence rate than using the LMS. However, using the LMS can achieve smaller steady-state MSD than using the NLMS at the same convergence rate.

${ }^{3}$ In this case, the LMS and NLMS have similar convergence rate. The LMS is preferred because of its lower complexity than the NLMS. Meanwhile, because of (39), the LMS may achieve lower state-state MSD than the NLMS at the same convergence rate.

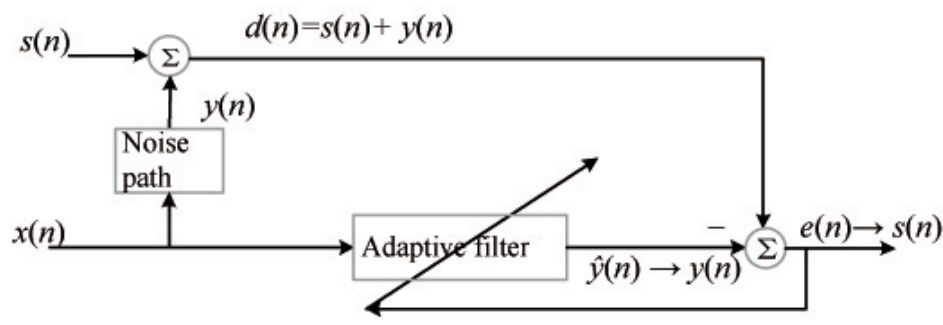

Fig. 1. Adaptive noise cancelling.

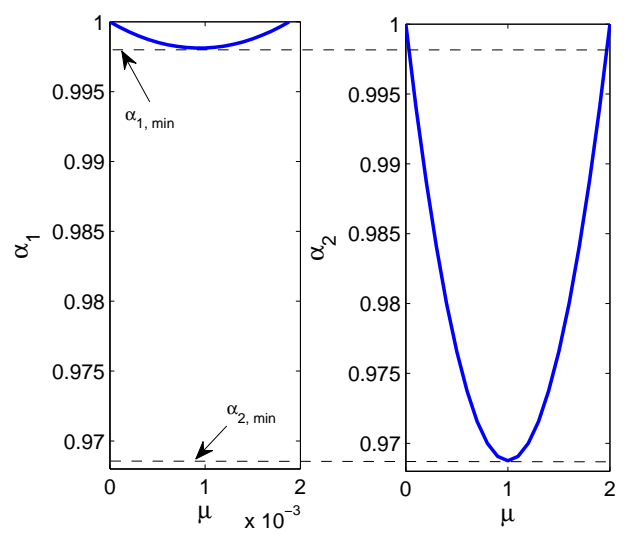

Fig. 2. Curves of $\alpha_{1}$ and $\alpha_{2}$ versus $\mu$, at $L=32$ and $\kappa=500$. 

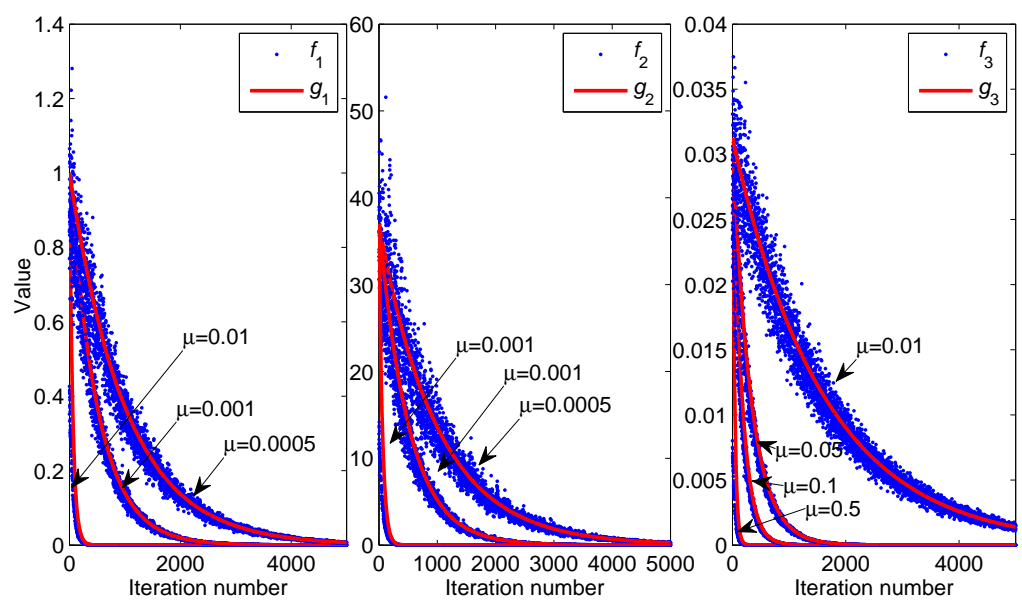

Fig. 3. Curves for (42)-(44) for the fixed noise path, where $L=32$ and the power of desired signals is $10^{-3}$. The six functions are $f_{1}=$ $E\left\{\mathbf{v}^{T}(n) \mathbf{x}(n) \mathbf{x}^{T}(n) \mathbf{v}(n)\right\}, g_{1}=\sigma_{x}^{2} \operatorname{MSD}(n), f_{2}=E\left\{\mathbf{x}^{T}(n) \mathbf{x}(n) \mathbf{x}^{T}(n) \mathbf{v}(n) \mathbf{v}^{T}(n) \mathbf{x}(n)\right\}, g_{2}=L \sigma_{x}^{4} \operatorname{MSD}(n)+(\kappa-1) \sigma_{x}^{4} \operatorname{MSD}(n), f_{3}=$ $E\left\{\frac{\mathbf{v}^{T}(n) \mathbf{X}(n) \mathbf{X}^{T}(n) \mathbf{v}(n)}{\mathbf{X}^{T}(n) \mathbf{X}(n)}\right\}$ and $g_{3}=\frac{1}{L} \operatorname{MSD}(n)$.


Fig. 4. Experimental and theoretical MSD curves of LMS with different reference inputs, where the desired signal $s(n)$ is a zero mean i.i.d. Gaussian sequence with variance $10^{-2}, L=32$. (a) The fixed noise path. (b) The time-varying noise path, $\sigma_{q}=10^{-4}$. 

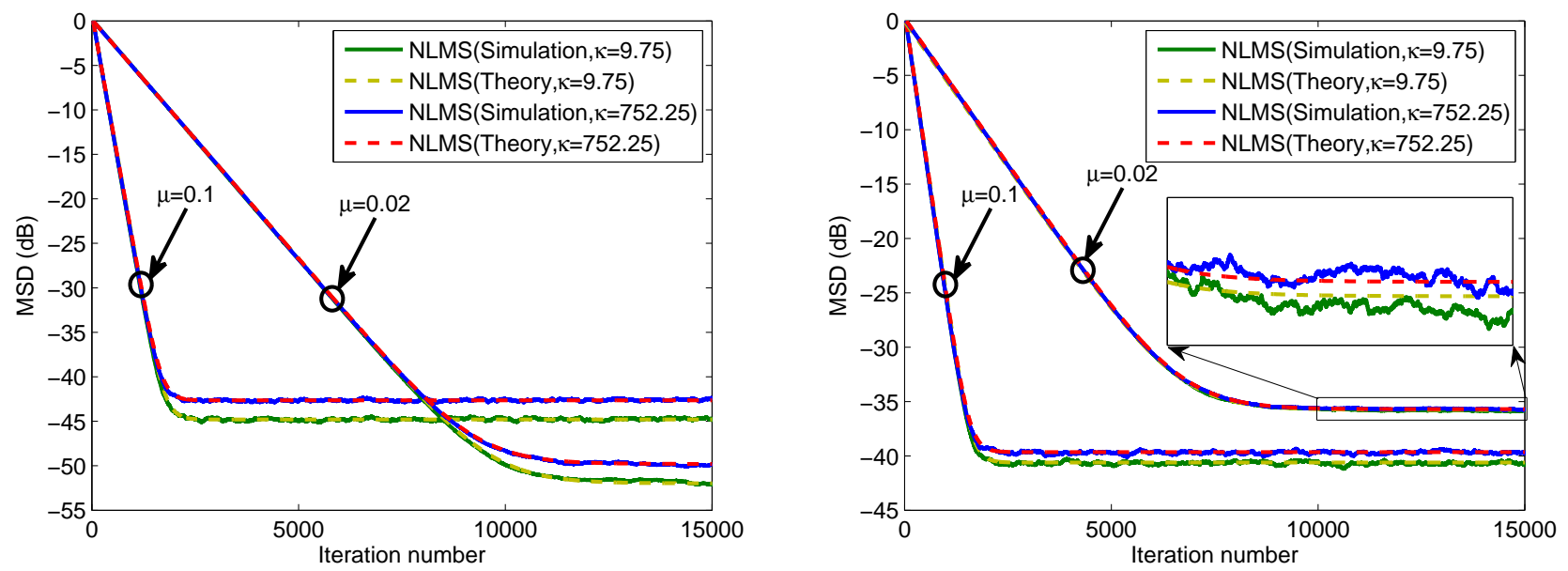

Fig. 5. Experimental and theoretical MSD curves of NLMS with different reference inputs, where the desired signal $s(n)$ is a zero mean i.i.d. Gaussian sequence with variance $10^{-3}, L=32$. (a) The fixed noise path. (b) The time-varying noise path, $\sigma_{q}=10^{-4}$.

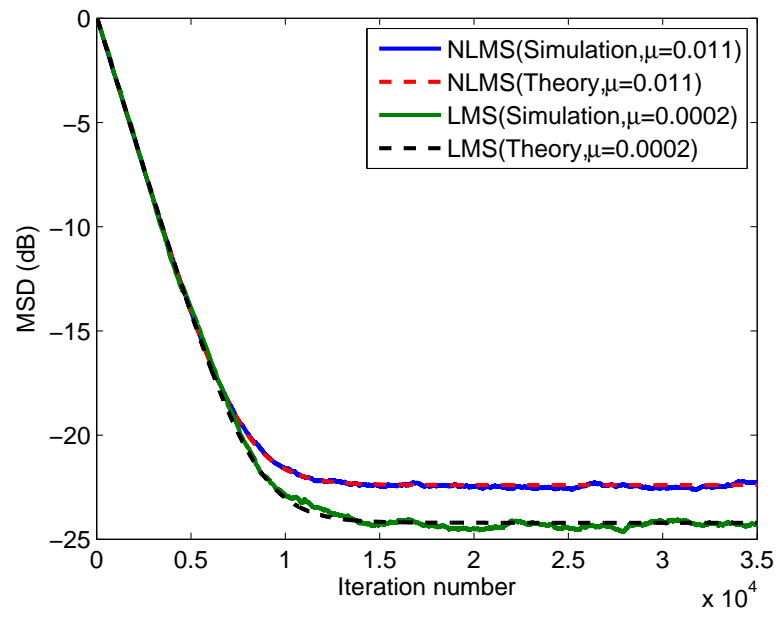

Fig. 6. Comparison of LMS and NLMS at same convergence rate for the fixed noise path, where $L=32, \sigma_{x}^{2}=2, \sigma_{s}^{2}=1$. 